\title{
OPTICALLY THIN GAS IN THE BLR OF SEYFERT GALAXIES
}

\author{
SIMON L. MORRIS \\ Mount Wilson and Las Campanas Obs. \\ 819 Santa Barbara St. \\ Pasadena, CA 91101 \\ and \\ MARTIN J. WARD \\ Institute of Astronomy \\ Madingley $R d$. \\ Cambridge CBS OHA \\ England
}

\section{Introduction}

Evidence has been accumulating that the gas which produces the broad line emission (BLR gas) in active galaxies has a range of physical conditions, and may indeed come from a distribution of spatially distinct regions (Gaskell 1982, Ulrich et al. 1984, Netzer 1987, Collin-Soufrin et al. 1988). In this paper, we try to separate out any contribution to the BLR emission line profiles from optically thin material. We do this by comparing the profiles of OI $\lambda 8446$ (a flourescence line only produced by clouds very optically thick to Lyman $\alpha$, Elitzur and Netzer 1985) and $\mathrm{H} \alpha$ (which can be produced by optically thick and optically thin clouds).

Observation were obtained at CTIO of 15 AGN, with a GEC CCD at a resolution of $4 \AA$ (FWHM), and were reduced in the usual manner (see Ward and Morris 1984). A polynomial fit was made to the underlying continuum and divided through, giving (after subtraction of 1.0) continuum subtracted line profiles. To overplot the line profiles of $\mathrm{OI}$ and $\mathrm{H} \alpha$ a scaling factor must be applied to one of the lines. In deriving this scaling factor, we assumed that at no point should the OI profile be significantly above the $\mathrm{H} \alpha$ profile.

\section{Results}

Of the 15 objects observed, 12 had strong enough OI for profile comparison to be made. 8 of these 12 objects showed no significant profile differences, 2 showed marginal differences, and 2 showed clear differences. The figure below shows the two objects with clear profile differences. 

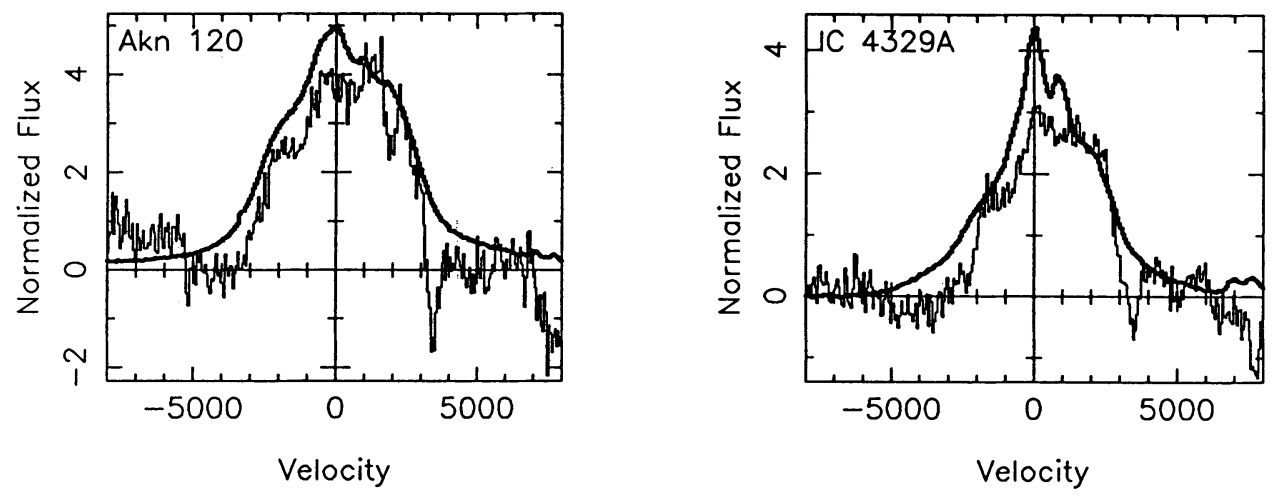

Figure 1 Profile comparisons for two AGN. Thick line: $\mathrm{H} \alpha$, thin line: OI $\lambda 8446$.

\section{Discussion}

The first point to make is that the majority of the objects observed show no profile differences. Thus either there is little optically thin material, or it shares the same velocity field as the optically thick material.

Both of the objects with clear profile differences show an excess of $\mathrm{H} \alpha$ relative to $\mathrm{OI}$ in the blue wing. Probably the simplest interpretation of this difference is that the emission comes from an optically thin outflow or wind, where the material on the far side of the nucleus is obscured.

What distinguishes these two objects from the rest of the sample? Both are known have variable line profile shapes (Wilson and Penston 1979, Peterson et al. 1983). Akn 120 has the highest $\mathrm{H} \alpha$ luminosity in our sample. IC $4329 \mathrm{~A}$ is heavily reddened, and after correction for this, also has a high $\mathrm{H} \alpha$ luminosity. Finally, in both objects, the line profiles are strongly red asymmetric. In order to determine which (if any) of these factors are important in producing the observed profile differences, a much larger sample of objects is needed. Some progress towards collecting this sample has been made this year using telescopes at Palomar and Las Campanas.

\section{References}

Collin-Souffrin, S., Dyson, J. E., McDowell, J. C. and Perry, J. J., 1988, M. N. R. A. S., $232,539$.

Elitzur. M. and Netzer, H., 1985, Ap. J., 291, 464.

Gaskell, C. M., 1982, Ap. J., 263, 79.

Netzer, H., 1987, M. N. R. A. S., 225, 55.

Peterson, B. M., Foltz, C. B., Miller, H. R., Wagner, R. M., Crenshaw, D. M. Meyers, K. A. and Byard, P. L., 1983, A. J., 88, 926.

Ulrich, M. H., Boksenberg, A., Bromage, G. E., Clavel, J., Elvius, A., Penston, M. V., Perola, G. E., Pettini, M., Snijders, M. A. J., Tanzi, E. G. and Tarenghi, M., 1984, M. N. R. A. S., 206, 221.

Ward, M. J. and Morris, S. L., 1984, M. N. R. A. S., 207, 867.

Wilson, A. S. and Penston, M. V. 1979, Ap. J., 232, 389 